\title{
Leishmania infantum LelF protein is an elF4A-like RNA helicase that modulates interleukin IL-12p70, IL-10 and TNF- $\alpha$ production in human monocytes
}

\author{
Mourad Barhoumi ${ }^{1 *}$, N Kyle Tanner ${ }^{2}$, Amel Garnaoui ${ }^{3}$, Josette Banroques ${ }^{2,4}$, Belhassen Kaabi ${ }^{1}$, Patrick Linder ${ }^{2}$, \\ Ikram Guizani?
}

From Institut Pasteur International Network Annual Scientific Meeting

Hong Kong. 22-23 November 2010

Leishmania LeIF antigen, homologous to eukaryotic initiation factor eIF4A, was originally described as a Th1-type natural adjuvant and as an antigen that induces an IL-12-mediated Th1 response in the peripheral blood mononuclear cells (PBMC) of leishmaniasis patients. We aimed at addressing the role of this protein and defining the minimum fragment necessary for inducing cytokine secretion. The study necessitated expression cloning of LeIF and 9 domains. Comparative biochemical and genetic analyses of LeIF and yeast eIF4A showed that LieIF is both an RNA-dependent ATPase and ATP-dependent RNA helicase in vitro and highlighted differences with yeast protein. In vivo experiments in yeast showed that LeIF cannot complement the deletion of the essential TIF1 and TIF2 genes in the yeast Saccharomyces cerevisiae that encode eIF4A. However, expression of LeIF results in a dominant negative phenotype, which is abolished by deletion of the most divergent $25 \mathrm{~N}$-terminal residues. LeIF is able to interact with yeast eIF4G; this suggested a role in the translation machinery. The assays measuring production of cytokines IL-12p70, IL-10 and TNF- $\alpha$ by PBMC-derived monocytes of healthy donors exposed to the different proteins showed that LieIF was able to induce the secretion of these cytokines. Unlike previous reports on LeIF from L. braziliensis and L. major, both amino and carboxyl parts of the protein were shown to induce the secretion of cytokines at significant levels. Our results suggest that this activity could be primarily located in amino acids 1-129 and 261-403. Furthermore, the induction of cytokines in monocytes of healthy subjects is not unique to the Leishmania protein. Indeed, 5 homologous proteins DEAD box in mammals and yeast were also able to induce the secretion of cytokines. This study confirms the importance of LeIF protein as a vaccine target and underscores its potential as drug target.

\section{Acknowledgements \\ Supported by MESRST-Tunisia (LROOSP04), UNICEF/UNDP/WorldBank/WHO special programme TDR (A30134).}

\section{Author details}

${ }^{1}$ Laboratoire d'Epidémiologie et d'Ecologie Parasitaire, Institut Pasteur de Tunis, 1002 Tunis-Belvedère, Tunisia. ${ }^{2}$ Département de Microbiologie et Médicine Moléculaire, Centre Médical Universitaire, Genève, Switzerland. ${ }^{3}$ Laboratoire d'Immunologie, Vaccinologie et Génétique Moléculaire, Institut Pasteur de Tunis, 1002 Tunis-Belvedère, Tunisia. ${ }^{4}$ Centre de Génétique Moléculaire, CNRS, Gif-sur-Yvette, France.

Published: 10 January 2011

\section{doi:10.1186/1753-6561-5-S1-P36}

Cite this article as: Barhoumi et al:: Leishmania infantum LelF protein is an elF4A-like RNA helicase that modulates interleukin IL-12p70, IL-10 and TNF- $\alpha$ production in human monocytes. BMC Proceedings 20115 (Suppl 1):P36.

\footnotetext{
* Correspondence: m1barhoumi@yahoo.com

'Laboratoire d'Epidémiologie et d'Ecologie Parasitaire, Institut Pasteur de

Tunis, 1002 Tunis-Belvedère, Tunisia

Full list of author information is available at the end of the article
} 